\title{
Experimental ulcerative herpetic keratitis. I. Systemic immune responses and resistance to corneal infection
}

\author{
CLARE CARTER ${ }^{1}$ AND D. L. EASTY ${ }^{2}$ \\ From the ${ }^{1}$ Department of Surgery, Medical School, University of Bristol, and \\ ${ }^{2}$ Bristol Eye Hospital, Lower Maudlin Street, Bristol BS1 2 LE
}

\begin{abstract}
SUMMARY The influence of previous infection with herpes simplex virus (HSV) on the susceptibility of rabbits to corneal inoculation of the virus was studied by means of the microtitration model for ulcerative herpetic keratitis. Systemic immune responses were assayed after skin and eye infections by means of the lymphocyte transformation (LT) and complement fixation (CF) tests. Both cutaneous and ocular primary infections resulted in cellular and humoral immune responses to HSV. In comparison to primary ocular infection, corneal disease in rabbits with previous skin infection ('secondary corneal infection') resulted in earlier initiation of cellular and humoral immune responses, while complement-fixing antibody titres reached a higher level. A previous cutaneous infection ('immunisation') provided considerable protection to the cornea and also accelerated recovery from corneal ulcerative disease. A correlation was observed between the initiation of the lymphocyte transformation response and the beginning of healing of corneal ulceration. A previous unilateral ocular infection induced an even higher degree of corneal resistance in that eye, and the opposite eye was protected to the same extent as by cutaneous immunisation.
\end{abstract}

The incidence of serum antibody to herpes simplex virus (HSV) in a mixed group of young adults was shown to be $65-69 \%,{ }^{1}$ and larger proportions of seropositive individuals have been found in some populations. ${ }^{2}$ Most of the general population is therefore thought to have experienced HSV infection, and the influence of immune responses to the virus on susceptibility to corneal infection is of interest.

Previous experimental work on specific immunity in relation to HSV infection of the cornea has demonstrated that an infection of one eye can protect that cornea from reinoculation of the virus. ${ }^{34}$ The extent of resistance shown by a previously uninfected eye after immunisation at a distant site was less clear. It was considered negligible by some authors, ${ }^{35}$ while others found evidence of some protection. ${ }^{67}$

In the present study we wished to determine whether HSV infection in the skin or eye would result, firstly, in detectable humoral and cellmediated immune responses, and, secondly, in corneal protection against subsequent infection.

Correspondence to Miss C. Carter.

\section{Material and methods}

The virus used was HSV-1, PH strain, with a stock titre of $4 \times 10^{8}$ (plaque-forming units) $\mathrm{PFU} / \mathrm{ml}$ in Vero cells. New Zealand White rabbits of weight 2 to $4 \mathrm{~kg}$ were used throughout.

Primary skin infection ('immunisation') was produced by injecting $12.5 \times 10^{5}$ PFU of virus intradermally in the right ear pinna at least 6 weeks before corneal inoculation of virus. Unilateral ocular infection was induced 8 weeks before bilateral challenge by the method of corneal inoculation of virus described below (with the range 3.9 to $15.6 \times$ $10^{5} \mathrm{PFU} / \mathrm{ml}$ virus).

\section{IMMUNE RESPONSES}

Lymphocyte transformation in response to HSV antigen (LT) and complement fixing (CF) antibody to HSV were measured in 6-7 and 2-4 rabbits respectively in each group.

The LT test was performed with cultures of whole blood. $0 \cdot 1 \mathrm{ml}$ peripheral blood (collected from a left ear vein) was cultured in $1 \mathrm{ml}$ Eagles minimum essential medium (MEM), with either $5 \mu \mathrm{g}$ PHA (purified phytohaemagglutinin, Well- 
come), 1:400 dilution of HSV antigen (cell associated antigen, supplied by Dr C. M. Bradstreet, Central Public Health Laboratory), 1:400 dilution of control antigen, or no stimulant. Cultures were set up in triplicate and incubated at $37^{\circ} \mathrm{C}, 5 \% \mathrm{CO}_{2}$, $100 \%$ humidity for 3 days, and $1 \mu \mathrm{Ci}{ }^{3} \mathrm{H}$-thymidine was added to each culture for the last 6 hours. Trichloracetic acid-insoluble (DNA-containing) material was recovered from each culture and the beta radioactivity counted as disintegrations per minute (dpm). Transformation indices (TI) were calculated as mean dpm of test cultures/mean dpm of controls.

\section{CORNEAL INFECTION}

The effect of previous cutaneous infection on corneal susceptibility to HSV was evaluated by comparing immunised and normal rabbits, while the effect of previous unilateral ocular infection was assessed by comparing corneal infection in previously infected, and the corresponding opposite, eyes. Four to five corneas were used in each group in each

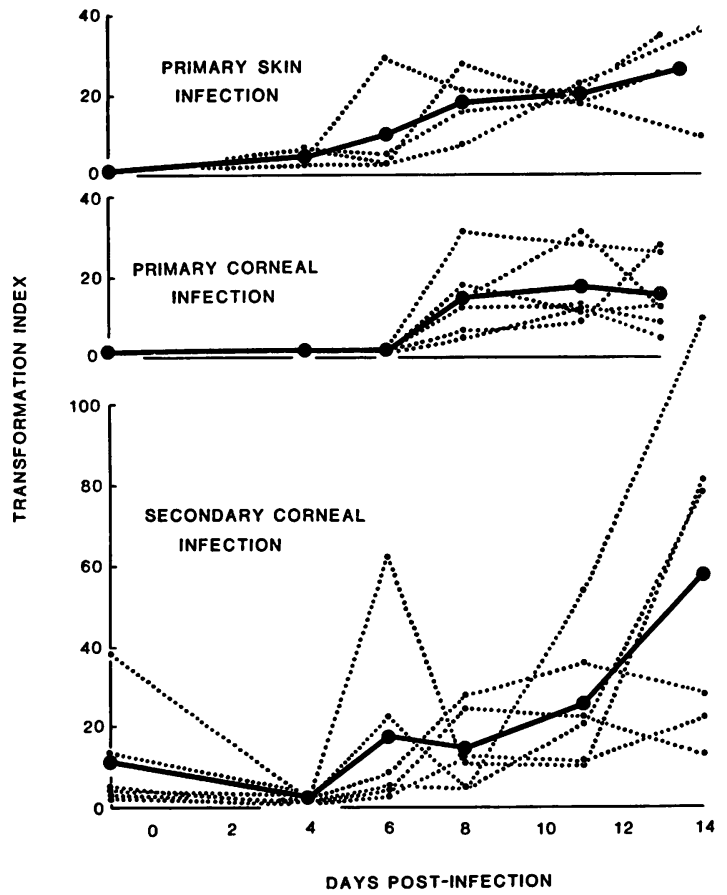

Fig. 1 Lymphocyte transformation in response to $H S V$ antigen, following primary skin, primary cornea, and secondary corneal infection. ........ Individual rabbits (triplicate cultures). _ Mean values. Note that the rise in transformation index occurred at day 8 following primary and day 6 following secondary corneal infection. experiment, and the accumulated data used to calculate CID $_{50}$ (corneal infectious dose for $50 \%$ of inoculations) values for primary and secondary corneal infection were derived from 50 corneas in all.

The method of corneal inoculation of virus was based on that of Jones and Al-Hussaini.8 Each cornea was inoculated at 16 sites by means of microtrephines -4 sites for each of 4 concentrations of virus. Serial 4-fold dilutions were used throughout, but the range of concentrations was altered to suit the experiment. A range of 1.0 to $62.5 \times 10^{5}$ or 3.9 to $250.0 \times 10^{5} \mathrm{PFU} / \mathrm{ml}$ was used in experiments to compare corneal susceptibility to primary and to secondary infection. To follow the progress and resolution of ulcerative disease ranges of 3.9 to $15.6 \times 10^{5}$ and 1.6 to $100.0 \times 10^{5} \mathrm{PFU} / \mathrm{ml}$ were used for primary and for secondary infections respectively.

The assessment of corneal infection was by 2 methods. Firstly, on day 2 after infection the presence or absence of ulcers at each site was recorded, and the percentages of infected sites for each dilution of virus inoculum were calculated for each treatment group of corneas. These data were used to produce a virus dose-response curve for each group and to calculate the dilution required to infect $50 \%$ of inoculated sites. ${ }^{89}$ Secondly, the extent of ulceration on each cornea was measured at intervals through the course of the disease by densitometry of accurate scale drawings. ${ }^{10}$

\section{Results}

IMMUNE RESPONSES

Both lymphocyte transformation (LT) and complement fixing (CF) antibody responses were seen following primary skin and primary and secondary corneal infections in all rabbits studied.

LT responses following primary skin infection were variable in timing, beginning between days 4 and 9 after infection. Primary corneal disease induced a rise in transformation index (TI) at day 8 , while secondary corneal infection resulted in an earlier increase, on day 6 (with one exception: p $<0.01$ by exact $2 \times 2$ test). The increase in TI was preceded by a decrease from the resting level 4 days after secondary corneal infection. The LT response subsequently followed a variable pattern in all 3 groups, either reaching a peak at 8 to 11 days or increasing until the experiment ended at day 13-14. There was a tendency for a higher TI to be reached following secondary than following primary corneal infection (Fig. 1). The lymphocyte transformation in PHA-stimulated cultures did not increase in response to any of the 3 types of infection. However, 

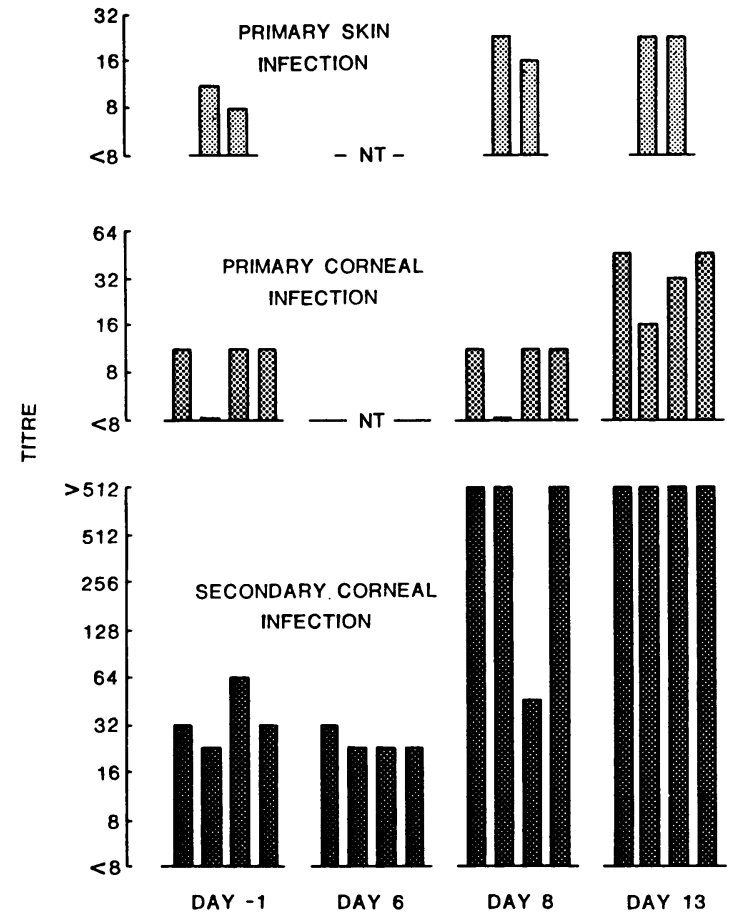

Fig. 2 Serum complement-fixing antibody titres following primary skin, primary corneal and secondary corneal infection. Note the lack of response at day 8 following primary and at day 6 following secondary infection.

a fall in this measurement was observed on day 4 in several rabbits with primary skin or corneal infections and in all rabbits undergoing secondary corneal infection.

Serum CF antibody to HSV was detected first at day 8-13 after primary skin infection and at day 13 after primary corneal inoculation of virus. After secondary corneal infection no antibody response was detected at day 6 , but a rise in titre occurred at day 8 , significantly earlier than after primary corneal infection $(\mathrm{p}<0.02$ by exact $2 \times 2$ test). The titres reached after the primary infections were similar, $1 / 24$ and 1/36 respectively, whereas a dramatic increase in titre to more than $1 / 512$ was observed following secondary corneal infection (Fig. 2).

\section{CORNEAL INFECTION}

The virus dose-response curves for primary and secondary corneal inoculation showed that a previous skin infection with the virus results in considerable protection of the cornea: CID $_{50}$ values were 3.33 and $87.51 \times 10^{5} \mathrm{PFU} / \mathrm{ml}$ for primary and secondary infection respectively. The differences in percentage infection were significant $(\mathrm{p}<0.001$ by exact $2 \times 2$ test) in each of 4 experiments. Following unilateral ocular infection, the opposite corneas of the rabbits were protected to a similar extent as those of immunised animals, while the previouslyinfected eyes exhibited a higher resistance $(p<0.05$ by exact $2 \times 2$ test) (Fig. 3 ).

The areas of corneal ulceration were greatly reduced in immunised animals. The mean areas of ulceration on day 2 were $155 \pm 24$ (SE) for primary and $14 \pm 4$ for secondary infection, and $298 \pm 47$ and $44 \pm 5$ on day 4 , after inoculation of both groups with 1.6 to $100.0 \times 10^{5} \mathrm{PFU} / \mathrm{ml}$ virus $(\mathrm{p}<0.025$ by rank-sum test). The progress of ulcerative disease showed an earlier beginning of healing, at day 6 , following secondary infection, compared to day 8 following primary infection $(\mathrm{p}<0.004$ by exact $2 \times 2$ test: Fig. 4). Corneas of previously infected eyes showed a very restricted degree of ulceration, which however persisted until day 8 or 11 . The extent of ulceration in the opposite corneas was similar to that of secondary corneal infection, while healing began on day 6 or 8 .

\section{Discussion}

The results of this study show, firstly, that both primary and secondary corneal HSV infection, as

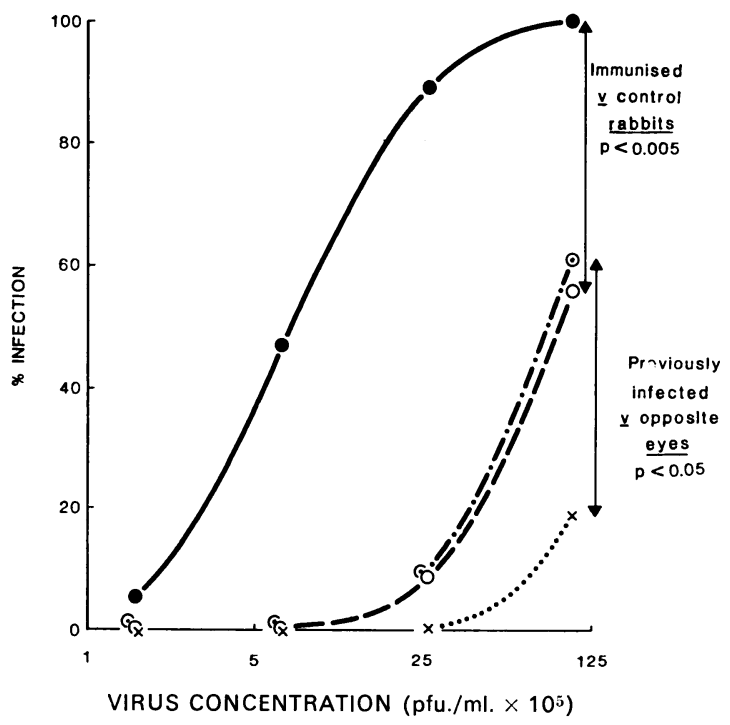

Fig. 3 Virus dose-response curves for

- primary corneal infection,

- - - secondary corneal infection,

$\odot \cdot-\cdot-\cdot-\odot$ previously infected corneas, and $+\cdots \cdots \cdots+$ opposite corneas of rabbits with a previous unilateral ocular infection. 


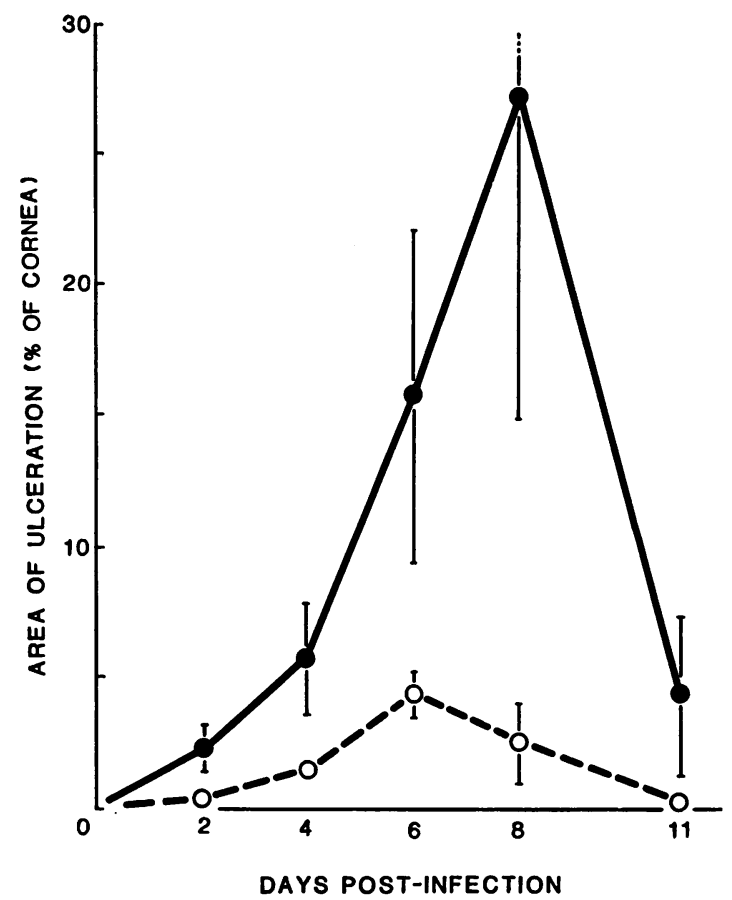

Fig. 4 Progress of ulcerative disease in primary

0 and secondary 0 - - - 0 corneal infection (mean areas of corneal ulceration \pm $S E$ ) following inoculation of respectively 15.6 to $3.9 \times$ $10^{5} \mathrm{PFU} / \mathrm{ml}$, and 100.0 to $1.6 \times 10^{5} \mathrm{PFU} / \mathrm{ml}$ of virus. Note that the initiation of the healing phase occurred at day 8 in primary and at day 6 in secondary corneal infection, coinciding in each case with the appearance of the $L T$ response (Fig. 1).

well as primary skin infection, induce systemic cellular and humoral immune responses to the virus. Secondly, a previous infection in the skin (or opposite eye) induces considerable corneal resistance to subsequent inoculation of the virus 6 weeks later. A previously infected eye shows even greater protection.

The patterns of the LT responses seen after primary and secondary corneal infection in rabbits were similar to those found in patients with primary or recurrent ulcerative herpetic keratitis. ${ }^{11}$ Since the experimental disease was produced by exogenous, not recurrent, infection, the decrease in LT levels for HSV and PHA seen in the early stage of corneal disease and similar observations by Cappe ${ }^{12}$ indicate that a fall in LT levels can be induced by proliferation of HSV. This suggests that similar findings in man prior to clinical recurrence might be related to the early stage of infection rather than to endogenous immunodeficits.

It should be remembered that systemic immune responses do not necessarily mirror the events occurring locally in the eye. However, it is of interest that the LT response and the healing phase of corneal ulceration were initiated simultaneously, at day 8 in normal rabbits and day 6 in immunised animals, while the CF antibody response did not appear until after the disease had begun to resolve. It is therefore possible that the activation of mechanisms operating locally to halt the spread of corneal infection may correlate with the development of the systemic LT response.

The increased protection observed in previously infected eyes could be due to specific immune processes, or alternatively, or in addition, to nonspecific effects of the earlier infection. The mechanisms which might be involved include antibody in the cornea ${ }^{5}$ or tears; increased numbers of HSV-immune or nonspecific lymphocytes, and leucocytes in the conjunctiva (and possibly the cornea); interferon; residual inflammatory changes.

This study of corneal infection in rabbits indicates that, whatever the immune mechanisms responsible, an individual with previous experience of the virus has a considerable degree of resistance to corneal HSV disease. The experimental observations would suggest that the severity of human ulcerative herpetic keratitis, and therefore probably the risk of virus spread into the stroma, may be reduced in the presence of immune responses to the virus and increased in cases of immunodeficiency.

Since most people have experienced clinical or subclinical HSV infection, we feel that ulcerative herpetic keratitis in rabbits with previous HSV infection provides a more accurate reflection of the human disease than does the corneal infection of normal rabbits. Although the Jones and Al-Hussaini model used in this study is not one of recurrence, the modification of this microtitration method to involve immunised rabbits should be useful for the evaluation of both topical and systemic agents in ulcerative herpetic keratitis, especially those inducing immunomodulation or immunosuppression.

We appreciate the help and encouragement of Professor J. H. Peacock and Dr M. O. Symes and the practical assistance given by Mr P. W. Madden. We are grateful to Dr C. M. Bradstreet for providing the herpes simplex antigen.

This work was kindly supported by a Research to Prevent Blindness grant from the RNIB.

\section{References}

1 Smith IW, Peutherer JF, MacCallum FO. The incidence of Herpesvirus hominis antibody in the population. J Hyg 1967; 65: 395-408.

2 Burnet FM, Williams SW. Herpes simplex: a new point of view. Med J Aust 1939; i: 637-42.

3 Hall RL, MacKneson RG, Ormsby HL. Studies of 
immunity in experimental herpetic keratitis in rabbits. Am J Ophthalmol 1955; 39: 226-33.

4 Okumoto M, Jawetz E, Sonne M. Studies on herpes simplex virus IX. Corneal responses to repeated inoculation with herpes simplex virus in rabbits. Am J Ophthalmol 1959; 47: 61-6.

5 Pollikoff R, Cannavale P, Dixon P. Herpes simplex virus infection in rabbit eye. Arch Ophthalmol 1972; 88: 52-7.

6 Amoils SP, Maier GMG. Treatment of severe herpes keratitis with cryosurgery and immunotherapy. Trans Ophthalmol Soc UK 1979; 99: 134-40.

7 Ey RC, Hughes WF, Holmes AW, Deinhardt F. The effect of I.D.U. on experimental and clinical herpes simplex infections. Trans Am Ophthalmol Soc 1963; 61: 100-7.
8 Jones BR, Al-Hussaini MK. Therapeutic consideration, in ocular vaccinia. Trans Ophthalmol Soc UK 1963; 83. 613-31.

9 Reed LJ, Muench H. A simple method of estimating fifty per cent end points. Am J Hyg 1938; 27 : 493-7.

10 Markham RHC, Carter C, Scobie M, Metcalf C, Easty DL. Double blind clinical trial of adenine arabinoside and idoxuridine in herpetic corneal ulcers. Trans Ophthalmol Soc UK 1977; 97 : 333-40.

11 Easty DL, Carter C, Funk A. Systemic immunity in herpetic keratitis. Br J Ophthalmol 1981; 65: 82-8.

12 Cappel R. Comparison of the humoral and cellular immune responses after immunisation with live, UV inactivated herpes simplex virus and a subunit vaccine and efficacy of these immunisations. Arch Virol 1976; 52: 29-35. 